\title{
Analysis for the Fault Caused by Two Different Formats of Bus Data Delivery
}

\author{
Rui Shen and Hengxin Cai \\ (A VIC Shenyang Aircraft corporation, Shenyang 110850, China)
}

\begin{abstract}
The electronic programmable controller of a fighter can receive the RS429 signal from atmospheric system, meanwhile it can transform the signal to RS422, but the indicator light indicates abnormal. The fault caused by the electronic programmable controller is found when it transforms the signal from the atmospheric system to the wrong format. The data formats of the RS429 and the RS422 are analyzed, the root cause and a solution to this problem are derived.
\end{abstract}

Keywords-electronic programmable controller; data transfer format; RS429; RS422

\section{INTRODUCTION}

When the electronic programmable controller of a fighter receives the atmosphere signal, the indicator light indicates abnormal. The data of static pressure and static temperature all show fault when receiving and reading the data form atmosphere by the ground test machines.

In order to identify the differences when the programmable controller is cross linked to the atmospheric parameters analog sources and atmospheric data machine respectively, and compare the RS429 data collected by atmospheric data machine tested by oscilloscope and from the standard analog signal source respectively, we found that except the different sending sequence sent by these two data source, other data all share the identical format. We send the data from the atmospheric parameters analog sources to the electronic programmable controller in the sequence which is identical to that sent by the atmospheric data machine, and it turns out that after receiving this data the calculation accomplished by the electronic programmable controller shows fault. This issue is same to that when the electronic programmable controller is cross-linked with the atmospheric data machine. Therefore we are sure that the changed data sequence of RS429 leads to the wrong processing result after the RS429 is received by the programmable controller and then transformed into RS422 data.

\section{THE StAndARD OF SERIAL INTERFACE RS429}

Adopting broadcast transmission principle, the RS429 data transmission transmitts in a manner of open-loop transmission with the bus data transfer rate of $12.5 \sim 100$ kbit / s. The parity check bit transmitted by a part of every digital makes it possible for the receiver to accompolish simple error checking. The bus has a strong anti-jamming capability, simple connection, high reliability, rich data resources, data precision and etc. And the transmission word is 32-bit.

RS429 data, a 32-bit data word, is regarded as a basic information unit and will be transmitted in pulses after modulation. These pulses are clssified into three levels_ the high level $(+10 \mathrm{~V})$, the medium level $(0 \mathrm{~V})$, and the low level $(-10 \mathrm{~V})$. The high level is identified as logic " 1 ", the low as logic " 0 " and the Medium level send their own clock pulse, separated by an interval (of four digits) word by word. And this interval will serve as word sync, as shown in Fig. 1.

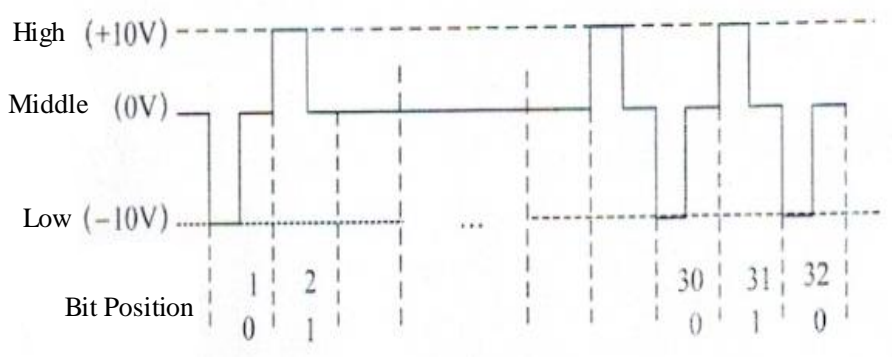

Figure 1. The schematic diagram of the signal RS429

In transmission, the basic information unit, the 32-bit data word, is divided into five application groups to send out, namely binary (BNR) data, two - decimal (BCD) data, discrete data, maintenance data and AIM data.

The sending data of the overall 32 data bits is delivered in an order of flag bit, source / destination flag bit, the data area, the symbol / status bits and parity bits. Where the flag bits and an the normal sequence are in opposite, a high level pulse will be sent out first and then a low one. And the cable transmission order is $8,7,6,5,4,3$, 2,1 .

According to the communication protocol of RS429 data bus, the communication between airborne equipments should first send data via a transmitter, put these data into ones that are in compliance with RS429 data bus specification for transmission on the data bus. When thses data reach the target device they will be transformed into a format which can be recogonized by the device through their receviors. According to the type, these data can be divided into serial data signal(32-bit), the control signal (read / write signal, chip select signal, parity and selection 
signals, and etc.), the clear signal, clock, and clock selectioon signal.

\section{THE STANDARD OF SERIAL INTERFACE RS422}

RS422 data singnals adopt a transmittion of differential transmission, which is also known as balanced transmission. It uses a twisted-pair which are idintidied as $A$ and $B$ respectively. Typically, the positive level between the transmission drivers $\mathrm{A}$ and $\mathrm{B}$ which is in +2 $\sim+6 \mathrm{~V}$ is regarded as a logic state, ther is also aonther siginal ground $\mathrm{C}$. The provinisions of the receiving end is opposed to that of the transmitting side, and the sending and receiving ends are connected to the corresponding AA and $\mathrm{BB}$ through a balanced twisted pair. But when the pulse between the recivers $A$ and $B$ is greater than +200 $\mathrm{mV}$, the output will be positive logic level, and when less than -200 , the output will be negative logic level. The level on the balanced line between recerivors always ranges from $200 \mathrm{mV}$ to $6 \mathrm{~V}$.

Serial communications can be divided into two types - the synchronous communication and the asynchronous communication.

When adopting synchronous communication, a number of characters are formed into the information group, so that characters can be transmitted one after another. But in the beginning of each set of information (usually called information frames) a synchronization character shall be added to notify the receiver when a valid data characters will arrive. And the data set can add a check character in the end. If there is no information to be transmitted, a null character shall be adde, as simultaneous transmission does not allow intervals.

The typical synchronous communication data format is shown in Fig. 2

When adopting the asynchronous communication, each character can randomly appear in the data stream, the transmission interval between two characters is arbitrary, so there should be some data bits before and after each character as separate position. The asynchronous communication is shown in Fig. 3. As shown in Fig. 3, in a standard format of asynchronous communication, when a charactor is in transmission, besides the actual transmission of the encoded information, a few more extra data bits shall also be transmitted. Specifically, before the transmission of a character, the output line must be in the "1" state, which is called identification state. At the begaining of the transmission, the output line will switch from the identicfication state to the " 0 " state as a start bit. From 5th to 8th bit after the start bit are the information bits. The information bits are arranged from low to high, the first one is the lowest bit character. In one delivery system, the number of information bits is fixed. The check bit is behind the information bit. The check bit can be set accordance with odd check,or parity check, and the check bit may not be set. The last data bit is "1" as the stop bit. in synchronization protocol, the BYN synchronization character can be used to achieve charactor synchronization between nodes, or to maintaine the synchronization in the absence of data transmission.

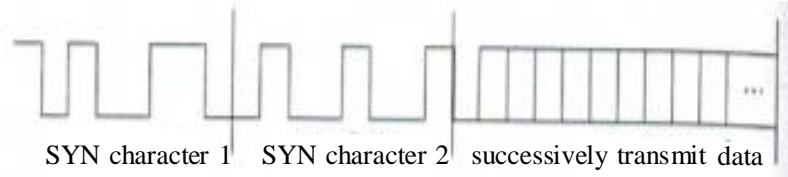

Figure 2. The typical data format of synchronous communication

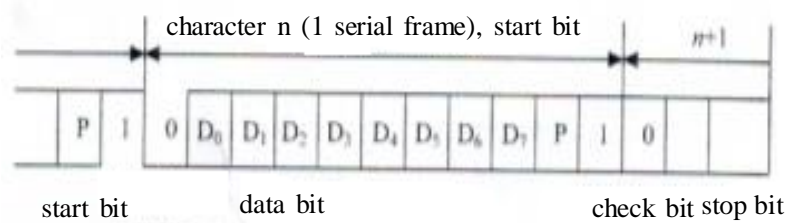

Figure 3. The typical data format of asynchronous communication

By comparison, when the transmission rate is identical, the information transmitted by synchronous communication mode is more efficient than asynchronous mode, since the proportion of non-data information in synchronous mode is relatively small. On the other hand, in synchronous mode, the two ends of the information transmission must be coordinated with the same clock. It is this clock that determines the position of each digit in the synchronous serial transmission.

\section{The Data Calculation and Decoding \\ PRINCIPLE IN ATMOSPHERIC DATA SYSTEM}

\section{A. The Data Reception Format of Atmospheric Data}

The RS429 signal output by the atmosphere computer is a digital code flow. Only when the numeric code format was tranformed into decimal form can they become acceptable and readable form during the process of the atmosphere data system tests.

\section{B. $\quad 3.2$ The Output Format of Atmosphere Data RS429}

The atmosphere data computer processes the pressure and temperature signals from the pressure sensors and temperature sensors, and output the atmosphere signal by RS429.

\section{FAULT ANALYSIS AND IMPROVEMENT MEASURES}

\section{A. Fault Analysis}

Programmable controller receives the atmosphere data on the machine by taking queries. It receives the RS429 data sent by the atmosphere machine in sequence cycle. In each cycle, when the programmable controller identifies the last received data as "P", it will package the received data into a set of RS422 data to send them in the data 
processing module for the calcalation. If the sequence of the data sent by atmosphere data machine is changed, which makes the final identification of data is not "P", then it will result in incomplete data receiving, some loss of data, billing errors, and clearing faults when the programmable controller receives and processes the atmosphere data.

Programmable controller receives the machine signal and indicates it by status indicators. When the atmosphere data machine sends RS429 data in certain cycle, programmable controller receives and process the software via RS429 signal, and convert it into RS422 signal data to transmmit it to the data processing module. The data processing module waits the uploaded RS422 signal by query for $6 \mathrm{~ms}$ (the reference data and the actual maybe inconsistent). If the aero signal is sent in certain fixed cycle and a fixed transmission sequence, then processing module will recieve complete signal receives data and the indicator will indifies normal. If the aero signal is sent in a changed cycle or a changed sequence, it may miss the $6 \mathrm{~ms}$-long waiting time and there will be lost data and the indicator will show abnormal indication in non-continuous fast strobe.

\section{B. Improvements}

Change the signal receiving and processing software in the programmable controller RS429, and improve signal integrity determination method of the receiver. We determine whether there were eight data received, instead of identifying the last data in the eight RS429 data. If there are eight data received, we will package these eight data in a set of RS422 data signal to transmit to the data processing module. Therefore, problem that the programmable controller receives incomplete data from the atmosphere data machine when the data sequence changes will be avoided and the fault that the clearing result shows abnormal when the programmable controller receives the atmosphere data will bw solved.

\section{VERIFY THE RESULTS}

Taking such measures, we change and improve the convertion of RS429 signals in the programmable controller and the RS422 signal receiving moudule and conduct the internal tests. After the changes are completed, the programmable controller and atmosphere data machine unit crosslinked and verified to ensure that the programmable controller works properly when receiveing the aero signals. We addressed the fault that the status indicator shows abnormal when the electronic programmable controller receives the atmosphere data signal when crosslinked to a certain airplane.

\section{REFERENCES}

[1] HB 6096 - 1986.SZ-01 Digital Information Transmission System [S] 1987.

[2] The United States] Texas Instruments Incorporated TI DSP / BIOS and Driver Development User Manual [M] Translated by Wang Junning, He Di, Ma Juan, Beijing: Tsinghua University Press, 2007.

[3] Li Rong, Liu Weiguo Aviation Design and Realization of ARINCA29 Bus Transceiver System [J]. Computer Measurement \& Control, 2005,13 (9): 46-48.

[4] Certain Air Data System Test Technical Report [R] Yantai: Naval Aeronautical Engineering Institute, 2000.

[5] Guo Enquan, Miao Sheng, Retrospect and Prospect of the Test Bus Development [J] Electronic Measurement and Instrument, 2009,23 (8): 1-7. 